\title{
Cognitive reserve and the severity of Alzheimer's disease
}

\author{
Reserva cognitiva e a severidade da doença de Alzheimer \\ Margarida Sobral1,2, Maria Helena Pestana ${ }^{2,3}$, Constança Paúl²
}

\begin{abstract}
Alzheimer's disease (AD) is a clinical syndrome caused by neurodegeneration and characterized by a progressive deterioration in cognitive ability and capacity for independent living. Cognitive reserve (CR) describes the capacity of the adult brain to cope with the effects of the neurodegenerative process and to minimize the clinical manifestation of pathology of dementia. The aim of this study was to evaluate the association of CR and the severity of AD. Method: This study was cross-sectional. Functional and neuropsychological abilities of 75 outpatients with probable AD diagnosis were evaluated. Patients completed two questionnaires, "Participation in leisure activities throughout life" and CR Questionnaire. Result: The relationship between Clinical Dementia Rating (CDR) and the level of CR was statistically significant (likelihood ratio (LR), $p=0.015$ ). Conclusion: The level of $C R$ influenced the severity of dementia. This study suggests that $A D$ patients with higher CR may benefit against cognitive decline after diagnosis of AD.
\end{abstract}

Keywords: Alzheimer's disease, cognitive reserve, education, leisure activities, lifelong occupation.

\section{RESUMO}

Doença de Alzheimer (DA) é uma síndrome neurodegenerativa caracterizada por uma deterioração progressiva das capacidades cognitivas e das competências para uma vida independente. A reserva cognitiva (RC) descreve a capacidade do cérebro adulto em lidar com os efeitos deste processo neurodegenerativo e minimizar a manifestação clínica da patologia de demência. 0 objetivo deste trabalho foi avaliar a associação entre RC e a severidade da DA. Método: Este estudo foi transversal. Foram avaliadas as competências funcionais e neuropsicológicas de 75 pacientes com diagnóstico provável de DA. Os pacientes completaram dois questionários, "Participação em atividades de lazer ao longo da vida" e o Questionário de RC. Resultados: A relação entre o nível de Clinical Dementia Rating (CDR) e de $R C$ foi estatisticamente significativa (likelihood ratio ( $L R$ ), $p=0,015$ ). Conclusão: 0 nível de RC influenciou a severidade da demência. Este estudo sugere que os doentes com DA com um maior nível de RC podem beneficiar de proteção relativamente ao declínio cognitivo após o diagnóstico de DA.

Palavras-chave: doença de Alzheimer, reserva cognitiva, educação, atividades de lazer, ocupação ao longo da vida.

Alzheimer's disease (AD) is the most common form of dementia in the elderly ${ }^{1}$ and a clinical syndrome caused by neurodegeneration and characterized by a progressive deterioration in cognitive ability and capacity for independent living. The course of $\mathrm{AD}$ is not the same in every person, but symptoms seem to develop over the same general stages. Cognitive reserve (CR) is a hypothetical construct that has been used to inform of cognitive aging and describes the capacity of the adult brain to deal with the effects of the neurodegenerative process and to minimize the clinical manifestation of pathology of dementia that is probably the result of innate intelligence or life experience ${ }^{2}$. The CR hypothesis suggests that individual differences in the ability to cope with $\mathrm{AD}$ pathology ${ }^{2,3}$ are consistent with the prediction that people with more reserve can cope with advancing $\mathrm{AD}$ pathology longer before it is expressed clinically ${ }^{3,4,5}$. CR is not fixed, but continues to evolve across the lifespan ${ }^{5}$. Variables pertaining to lifetime experience (education, occupation attainment and leisure activities $)^{4,5}$ are the most commonly used proxies for $\mathrm{CR}$ and help retain cognitive function in old age. Epidemiological studies suggest that lifelong experiences, including educational, occupational attainment and leisure activities (LA) in later life, can increase reserve ${ }^{4}$. Greater understanding of the concept of CR could lead to

${ }^{1}$ Hospital de Magalhães Lemos, Serviço de Psicogeriatria, Porto, Portugal;

${ }^{2}$ Universidade do Porto, Instituto de Ciências Biomédicas Abel Salazar, Unidade de Investigação e Formação sobre Adultos e Idosos, Porto, Portugal; ${ }^{3}$ Instituto Universitário de Lisboa, Instituto Superior de Ciências do Trabalho e da Empresa, Lisboa, Portugal.

Correspondence: Margarida Sobral; Hospital de Magalhães Lemos, Serviço de Psicogeriatria; Rua do Professor Álvaro Rodrigues, 4149 - 003; Porto, Portugal; E-mail: margaridasobral@hmlemos.min-saude.pt

Conflict of interest: There is no conflict of interest to declare.

Received 18 May 2014; Received in final form 02 January 2015; Accepted 24 January 2015. 
interventions to slow cognitive aging ${ }^{4,6}$. Sobral and Paúl ${ }^{7}$ demonstrated that $\mathrm{AD}$ patients with higher levels of education achieved better results on cognitive tests and the participants with higher participation in leisure activities (PLA) exhibited better results on cognitive and functional tests than those with lower participation.

Tschanz et al. ${ }^{8}$ found that a significant proportion of persons with $\mathrm{AD}$ progresses slowly and suggest ongoing need to identify factors that may slow the progression of $\mathrm{AD}$. Other studies have noted faster rates of progression in high $\mathrm{CR}$ patients after the diagnosis of $\mathrm{AD}^{3,9,10}$. A research has indicated that high PLA is associated with more rapid cognitive decline than in those with lower PLA ${ }^{9}$ and some studies have shown that $\mathrm{AD}$ patients with higher education have a more rapid cognitive decline ${ }^{3,10}$ than those with lower education, because at any level of clinical severity, the underlying progression is more advanced in patients with higher education, which has been attributed to harbouring a higher pathological burden at the time of clinical dementia for subjects with higher education ${ }^{3}$. Other authors ${ }^{7,11}$ concluded that education slows the rate of cognitive decline in person with $\mathrm{AD}$ and Treiber and colleagues ${ }^{12}$ proved that the increased engagement in cognitive LA through late life was associated with slower deterioration in general cognitive ability in mild dementia. Sobral and Paúl ${ }^{7}$ found that $\mathrm{AD}$ patients with high education and high PLA may benefit from a slower cognitive and functional decline after diagnosis of $\mathrm{AD}$. Other studies have found no relationship between education and cognitive decline in the clinical course of $\mathrm{AD}^{13}$ and between occupation and cognitive decline in the clinical course of $\mathrm{AD}^{11}$.

We wanted to know the association between CR and disease progression and the aim of this study was to evaluate the association of $\mathrm{CR}$ and the severity of $\mathrm{AD}$.

\section{METHODS}

\section{Participants}

This study includes 75 outpatients (mean age: 80.20 years; range 61-92 years) diagnosed with probable $\mathrm{AD}$, recruited at a psychiatric hospital, psychogeriatrics service. This Psychogeriatrics Service is designed to follow-up patients with dementia, using an integrated multidisciplinary approach to diagnosing and managing dementia. Physical, neurological, neuropsychological and psychiatric examinations, neuroimaging and additional tests, including blood tests were used to triage the various types of dementia. Diagnosis of $\mathrm{AD}$ among the individuals selected was obtained according to criteria of the Diagnostic and Statistical Manual of Mental Disorders, $5^{\text {th }}$ edition ${ }^{14}$ and the National Institute on Aging and the Alzheimer's Association workgroup for probable $\mathrm{AD}^{15}$. We excluded participants with mild cognitive impairment and psychiatric disorders. All participants were free of severe medical conditions other than those pertinent to the study, including no history of alcohol abuse or recurrent substance abuse or dependence and no history of heart disease or diabetes. The patients (or his/her legal representative) included in the study have provided written consent to participate in this study.

\section{Instruments}

In order to quantify $\mathrm{CR}$, the Cognitive Reserve Questionnaire (CRQ) ${ }^{16}$ was administered. The CRQ includes the participation in as activities (reading, intellectual games), years of education, years of parents' education, occupation, training courses, musical training and languages carried out by participants during their adult lifetime. The CRQ has been validated for the Portuguese population and CRQ has high reliability and suitable content validity $^{17}$. CRQ consists of eight items and the Portuguese version of CRQ proved to be a valuable CR instrument for testing in research and clinical setting ${ }^{17}$. Regarding reliability the internal consistency of CRQ was 0.795 (Cronbach's Alpha). The factorial analysis of principal components with varimax rotation shows good indicators of the quality of the adjustment obtained either by Goodness of fit index $=0.99$ and by Root Mean Square Residual $=0.048$, which were based on the residual matrix correlation assuring the validity of the construct. The existence of eigenvalues $>1$ (Kaiser Criterion) and the steepening of the scree plot indicate the existence of a single dimension in CR. The responses were consistent, because there was a good correlation between the items, according to the measure of sampling adequacy Kaiser-Meyer-Olkin $=0.761$ and Bartlett's Sphericity Test $=204.159 ; \mathrm{df}=28 ; \mathrm{p}<0.01$.

Data on PLA was obtained from a questionnaire, "PLA throughout life" , answered by the participants or/and the caregiver. We begin by discriminating current and past activities. The caregivers were asked to confirm the information about the PLA throughout life of patients as current low PLA of patients may be a consequence of cognitive decline. This tool includes mental activities (reading books/newspapers, jigsaw puzzles), physical activities (walking or other sport), social activities (playing cards/board games, visiting friends or relatives), productive activities (housekeeping, babysitting, gardening, crocheting) and recreational activities (listening to the radio, watching television). Participants were asked it they regularly engaged in other particular activities, to specify which types of activities they were engaged in and to report the frequency of PLA throughout life. The instrument presents a global score and classifies people in three categories (low, medium and high PLA throughout life). The questionnaire "PLA throughout life", has 17 questions and for each question, subjects received 5 points for "daily", 4 points for "several days per week", 3 points for "once a weekly", 2 points for "two or three days per month", 1 points for "monthly" and 0 points for "never or less than a once a month". Its quotation 
can vary from 0 points up to 85 points. The factor structure of an instrument can also be used to assess the structural validity of the observe scores. We conducted an exploratory factor analysis using varimax rotation. Through the analysis of major components 5 factors were extracted. The total variance explained by the questionnaire is of $55.3 \%$ and the KMO value of sample adequacy is 0.6. The variance explained by each factor was $1^{\circ}$ factor (cognitive LA) $=19.5 \%$ (Cronbach's Alpha $=0.7), 2^{\circ}$ factor $($ productive and social LA) $=11.6 \%$ (Cronbach's Alpha $=0.6$ ) and other factors (unnamed because they have a Cronbach's Alpha $<0.5)$ : $3^{\circ}$ factor $=9.2 \%$, $4^{\circ}$ factor $=7.7 \%$ and $5^{\circ}$ factor $=7.3 \%$.

All participants were given the Mini-Mental State Examination (MMSE) ${ }^{18}$ for cognitive screening, as well as the Clinical Dementia Rating (CDR) which classifies dementia along 3 stages of severity as a function of overall cognitive and functional impairment. We applied the Portuguese version of the MMSE from Guerreiro et al. ${ }^{19}$. The MMSE includes questions on orientation, registration, attention and calculation, recall, language and visual construction. The normative cut-off values adjusted to the education for Portuguese population were used. The cut-off points for the diagnosis of dementia are above 27 if they had $>11$ years of education, $\leq 22$ if they had 1-11 years of education or $\leq 15$ if they were illiterate. Despite some limitations, MMSE score is a good indicator of severity of the disease. $\mathrm{CDR}^{20}$ classifies dementia along 3 stages of severity as a function of overall cognitive and functional impairment. We used the translation and adaptation of Garret et al. ${ }^{21}$. The CDR determines the impairment associated with dementia, through parameters such as memory, orientation, judgment and problem solving, community affairs, home and hobbies and personal care. The overall CDR score is obtained by a standard algorithm to stage the patient's level of impairment: 0 (no impairment), 0.5 (very mild impairment), 1 (mild dementia), 2 (moderate dementia), and 3 (severe dementia).

Addenbrooke's Cognitive Examination Revised (ACE-R) ${ }^{22}$ is a brief cognitive test that assesses five cognitive domains, namely attention/orientation, memory, verbal fluency, language and visuospatial abilities. Total score is 100, higher scores indicates better cognitive functioning. The ACE-R has been validated for the Portuguese population ${ }^{23}$.

Functional abilities were assessed with two activities of daily living scales: the Barthel's Index ${ }^{24}(\mathrm{BI})$, addressing basic activities of daily living such as grooming, eating, bathing, dressing, mobility, and the Lawton and Brody's Index ${ }^{25}$ (LBI), targeting instrumental activities (e.g. managing money, using the telephone). The BI have a possible total scores range from 0 to 100 (fully independent) and the LBI have a possible total scores range from 8 (independent) to 30 (completely dependent). Socioeconomic status was evaluated according to Graffar Index ${ }^{26}$. This index has five categories from $\mathrm{I}$ (the highest) to $\mathrm{V}$ (the lowest). The lifelong occupation was assessed with "National Classification of Occupations"27.

\section{Procedure and statistical design}

This study was cross-sectional and used data from the database of a research project designed to investigate $\mathrm{CR}$ and $\mathrm{AD}$ among patients recruited at a mental health hospital. The research protocol was approved by the Review Board (ethic committee) of the institution where the study was conducted. Data collected included socio-demographic and clinical variables including age, gender, marital status and retirement status and variables that usually inform CR (education level, lifelong occupation and frequency of PLA). Each participant underwent a standard evaluation, including medical history, physical examination, laboratory tests and a neuro-imaging (computed axial tomography). $\mathrm{AD}$ patients were evaluated with MMSE and CDR for recruitment at the first consultation of multidisciplinary assessment. Patients were submitted to a functional and neuropsychological evaluation. We have examined specific neuropsychological domains such as memory, language, attention, visuospatial ability and executive functioning. We have rated the severity of dementia according to the CDR.

In this study variables hypothesized to represent the CR construct were: CRQ (level of CR), education (as defined in terms of level of educational attainment), frequency of PLA (scores of LA) and lifelong occupation. General exploratory analyses were conducted to determine sample characteristics. For statistical analysis we computed descriptive statistics with percentages, mean, trends, standard deviations and percentiles. In order to inferential relationship between the metric and categorical variables with two or more categories we used the Student t-test and F test and Brown-Forsythe's One-Way ANOVA; to check the relationship between categorical variables we used whenever there were no interactive effects, models of contingency tables, using the Chi-square test Pearson, Fisher and likelihood ratio (LR), depending on the number of categories for analysis; the relationship between categorical variables was analyzed by using the interactive effects logistic regression models and relative risk to quantify the effects, the degree of association between variables was evaluated using metrics R Pearson ${ }^{28}$. The analyses were conducted using the IBM Statistical Package for Social Sciences 21 .

\section{RESULTS}

Table shows the sociodemographic and clinical characteristics of $\mathrm{AD}$ participants.

There was a significant majority of women compared to men, according to the binomial test $(\mathrm{p}<0.001)$. The age difference between the sexes was not statistically significant. It was more likely to find married men (80.5\%) than married women $(30.9 \%)$, likelihood ratio test $(p<0.001)$, while in other marital status differences were not relevant. Women had very different levels of education. The mean 5.75 years 
Table. Demographic and clinical characteristics.

\begin{tabular}{|c|c|c|}
\hline Demographic and clinical characteristics & AD patients $(n=75)$ & Tests (p) \\
\hline $\operatorname{Men}(\%)(n)<$ Women (\%) (n) & $26.7(20)<73.3(55)$ & Binomial $(p<0.001)$ \\
\hline Age (mean) (SD) (Min/Max) & $80.20(5.64)(61 / 92)$ & t-test $(p=0.3)$ \\
\hline Married (\%) (n) & $45.3(34)$ & \\
\hline Widower (\%) (n) & $38.7(29)$ & $\operatorname{LR}(p=0.6)$ \\
\hline Single (\%) (n) & $10.7(8)$ & $\operatorname{LR}(p=0.6)$ \\
\hline Divorced (\%) (n) & $5.3(4)$ & $\operatorname{LR}(p=0.6)$ \\
\hline Educacional level (5\% trimmed mean) (IR) (Min/Max) & $5.45(3 / 9)(0 / 17)$ & \\
\hline \multirow[t]{2}{*}{ Men $>$ Women } & $7.39(4 / 10)(2 / 17)>$ & \\
\hline & $4.76(3 / 4.5)(0 / 16)$ & t-test $(p=0.025)$ \\
\hline Iliterate (\%) (n) & $6.7(5)$ & \\
\hline Reading and writing (\%) (n) & $22.7(17)$ & \\
\hline 4 years $(\%)(n)$ & $41.3(31)$ & \\
\hline$>4-9$ years $(\%)(n)$ & $16.0(12)$ & \\
\hline$\geq 11$ years $(\%)(n)$ & $13.4(10)$ & \\
\hline Portuguese nationality (\%) (n) & $100(75)$ & \\
\hline Work Retired (\%) (n) & $96(72)$ & \\
\hline Unskilled workers & $57.3(43)$ & \\
\hline Men $<$ Women & $20 \%<70.9 \%$ & $\operatorname{LR}(p<0.001)$ \\
\hline Skilled workers & $10.7(8)$ & $\operatorname{LR}(p=0.4)$ \\
\hline Service workers, safety, vendors and administrative staff & $24.0(18)$ & $\operatorname{LR}(p=0.4)$ \\
\hline Intermediate level professions, intellectual and scientific activity & $8.0(6)$ & $\operatorname{LR}(p=0.4)$ \\
\hline Years of disease (mean) (SD) (Min/Max) & $5.17(3.31)(1 / 13)$ & t-test $(p=0.223)$ \\
\hline MMSE (mean) (SD) & $17.15(4.57)$ & t-test $(p=0.000)$ \\
\hline ACE- $\mathrm{R}$ & $86.66(18.64)$ & t-test $(p=0.000)$ \\
\hline Barthel Index & $23.36(5.18)$ & t-test $(p=0.000)$ \\
\hline Lawton Index & $41.53(15.60)$ & t-test $(p=0.000)$ \\
\hline Social Class (Graffar) (\%) (n) & & $\operatorname{LR}(p=0.241)$ \\
\hline II (medium/high) & $10.7(8)$ & \\
\hline III (medium) & $42.7(32)$ & \\
\hline IV (medium/low) and V (low) & $46.7(35)$ & \\
\hline $\mathrm{CDR}=1(\%)(\mathrm{n})$ & $46.7(35)$ & $\operatorname{LR}(p=0.463)$ \\
\hline $\mathrm{CDR}=2(\%)(n)$ & $36.0(27)$ & \\
\hline $\operatorname{CDR}=3(\%)(n)$ & $17.3(13)$ & \\
\hline
\end{tabular}

of education was replaced by a trimmed mean of $5 \%$ (5\% trimmed mean $=5.45$ ), while the standard deviation was replaced by the interquartile range Tukeys Hinges where 50\% of men had between 4 and 10 years instruction, whereas $50 \%$ of women had between 3 and 4.5 years. The men had on average 2.63 more years of education than women. For each increase of one category of education, it was $13 \%$ more likely to be male than female, as binary logistic regression model, with good sampling adequacy (Hosmer and Lemeshow test $=1.73$, $\mathrm{df}=4, \mathrm{p}=0.79)$ and $\operatorname{Exp}(\mathrm{sex})=1.13$. It was more likely to find women with no qualifications $(70.9 \%)$ than men $(20 \%)$, according the likelihood ratio test (LR) $(p<0.001)$, whereas in the other categories the differences were not significant. The difference of years of disease between the sexes was not statistically significant. The difference of social classes between the sexes was not statistically significant.

The patients with "> 4 years of education" obtained a mean of 19.21 points on the MMSE, while the illiterate obtained a mean of 9.60 points. The mean of ACE-R in elderly with "> 4 years of education" was 49.83, whereas the mean of ACE-R in older persons without any educational level was 15.60. The BI mean of the participants with " $>4$ years of education" was 86.46, whereas the $\mathrm{BI}$ mean of illiterate $\mathrm{AD}$ patients was 70.00 . The LBI mean of illiterate patients was 27.80 and with "> 4 years of education" group was 22.38. The patients with " $\geq 36$ scores" on "PLA throughout life" questionnaire obtained on 19.62 of mean in the MMSE, while the patients with " $\leq 25$ scores" obtained 17.00. The mean in ACE-R of elderly with " $\geq 36$ scores" on "PLA throughout life" questionnaire was 52.95 , while in the " $\leq 25$ scores" group obtained 37.38. The MMSE mean of "Intermediate level professions, intellectual and scientific activity" group was 19.83, while the MMSE mean of "Unskilled workers" group was 15.98 . The mean in ACE-R of "Professions intermediate level, intellectual and scientific activity" group was 49.00, while in the “Unskilled workers" group was 37.35. 
Association of cognitive reserve and the severity of Alzheimer's disease

Regarding the association of cognitive measures and the severity of $\mathrm{AD}$, there was a statistically significant decreasing linear trend between MMSE and CDR, ANOVA $(\mathrm{p}<0.001)$.

The Figure 1A shows the patterns of MMSE obtained for different $C R$ levels by $C D R$ and the Figure $1 B$ shows the patterns of ACE-R obtained for different CR levels by CDR. The "medium/high level of CR" group (more or equal than 7 points on CRQ) obtained the higher scores on MMSE and ACE-R (see Figures $1 \mathrm{~A}$ and $1 \mathrm{~B})$. The participants with higher CR levels had higher scores on cognitive tests than elderly with lower CR levels. The Figure $1 \mathrm{C}$ show patterns of BI obtained for different CR levels by CDR. The "low level of CR" group obtained the higher scores on $\mathrm{BI}$. $\mathrm{AD}$ patients with higher levels of $\mathrm{CR}$ showed worse results with a BI than those with lower levels of CR.

The Figure 2A shows the patterns of PLA for different CR levels by CDR. The participants with "medium/high level of CR" group had the higher scores on PLA. The Figure 2B shows the patterns of MMSE obtained for different education levels by CDR. This study showed that AD patients with higher levels of education achieved better results on MMSE.

As standardized adjusted residual $=2.1$, only elderly with more than five years of education were more likely to have mild dementia than moderate or severe dementia ( $45.7 \%$ vs $33.3 \%)$. Up to 4 years of education the CDR patients do not differ from each other. As standardized adjusted residual $=2.0$, only "Service workers, safety, vendors and administrative staff" are more likely ( $34.3 \%$ vs $24 \%$ ) to have had mild stage dementia than in moderate or severe dementia.

The relationship between the CR (evaluated with the $\mathrm{CRQ}$ ) and the years of evolution of dementia along the CDR, showed no statistically significant differences, according to Chi-Square Test $(p=0.946)$. The results showed that the level of CR did not influence the number of years of disease evolution. We crossed the disease stage (assessed with the CDR) and the years of the disease and we found statistically significant differences only in the category of the mild dementia, according to Chi-Square Test ( $p<0.001)$. The years of the disease were associated with the severity of the disease. The relationship between CDR and the level of CR (assessed with the CRQ) was statistically significant (LR, $p=0.015$ ). Decomposition of this test shows that mild dementia presents values statistically lower than those with moderate or severe dementia (LR, $p=0.027$ ). The CDR moderate and severe does not distinguish from each other in the CRQ. The level of CR influenced the severity of dementia (severity of dementia assessed with CDR).

\section{DISCUSSION}

According the current literature, $\mathrm{AD}$ is characterized by inexorably progressive deterioration in cognitive ability and
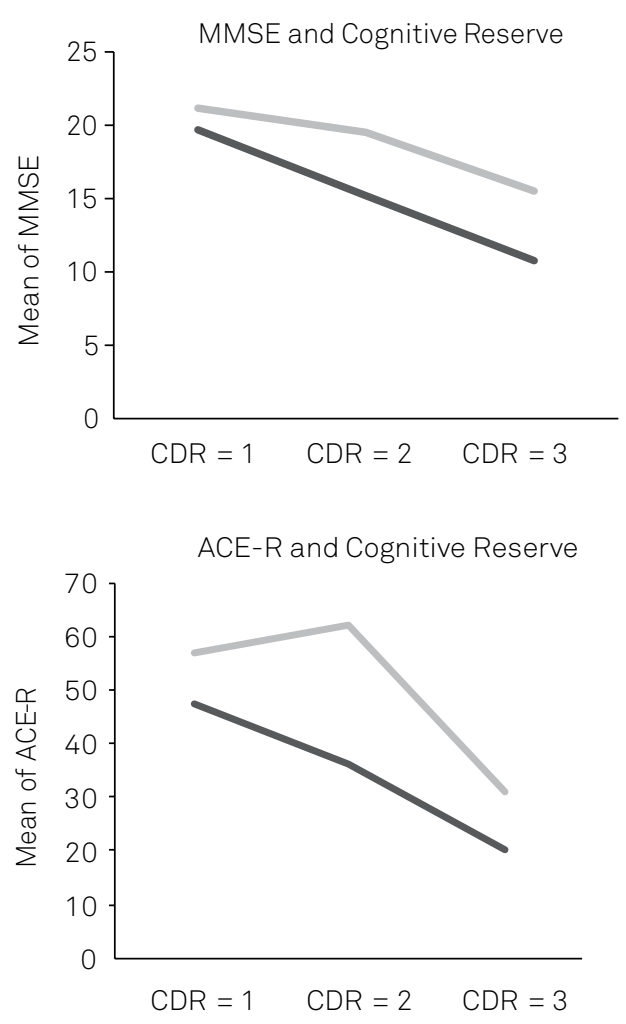

Barthel Index and Cognitive Reserve

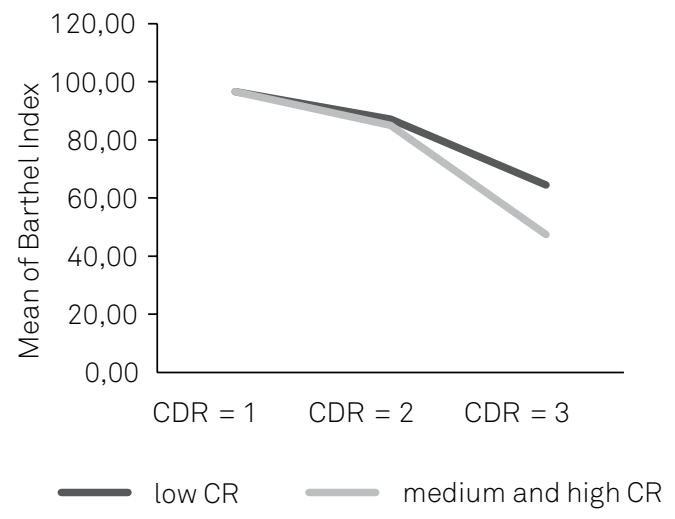

CR: Cognitive Reserve; MMSE: Mini-Mental State Examination; ACE-R: Addenbrooke's Cognitive Examination Revised; CDR: Clinical Dementia Rating; $\mathrm{CDR}=1$ : mild dementia; $\mathrm{CDR}=2$ : moderate dementia; $\mathrm{CDR}=3$ : severe dementia .

Figure 1. Patterns of MMSE, ACE-R and Barthel Index obtained for different CR levels. (A) Patterns of MMSE obtained for different CR levels (Cognitive Reserve Questionnaire); (B) Patterns of ACE-R obtained for different CR levels (Cognitive Reserve Questionnaire); (C) Patterns of Barthel Index for different CR levels.

capacity for independent living ${ }^{29}$ and, in this study we evaluated the association of $\mathrm{CR}$ and the severity of $\mathrm{AD}$ in an outpatient sample. We noticed a relationship between the level of $\mathrm{CR}$ and the level of staging of dementia (severity of dementia assessed with CDR). So, the level of CR influenced the severity of dementia. Education, occupation attainment and LA are the most commonly used proxies for CR and our study was consistent with other studies which showed that high 
Participation in leisure activities and Cognitive Reserve

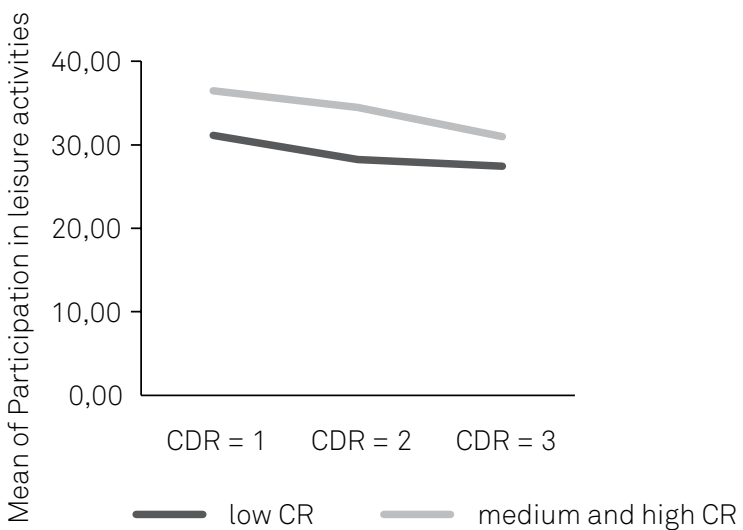

MMSE and Education

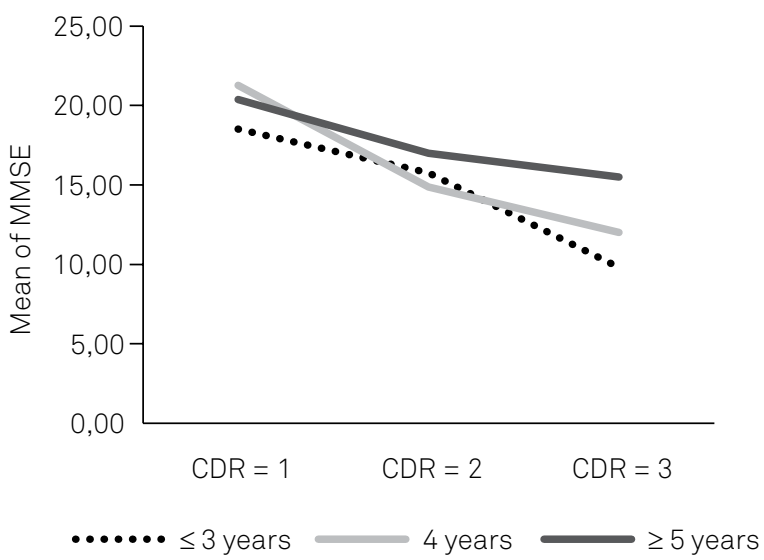

CR: Cognitive Reserve; MMSE: Mini-Mental State Examination; CDR: Clinical Dementia Rating; $C D R=1$ : mild dementia; $C D R=2$ : moderate dementia; $\mathrm{CDR}=3$ : severe dementia; " $\leq 3$ years": Three years or less than three years of education; "4 years": 4 years of education; " $\geq 5$ years": Five years or more than five years of education.

Figure 2. Participation in leisure activities and CR; MMSE and education. (A) Patterns of participation in leisure activities for different CR levels by CDR. (B) MMSE and Education.

levels of CR, education, high PLA and substantively complex work (eg, "Intermediate level professions, intellectual and scientific activity") influenced the rate of cognitive decline in persons with $\mathrm{AD}^{7,11,12}$.

The results of this study confirm other studies' results have shown that higher levels of education are associated with better performance on cognitive tests ${ }^{7,30}$. The group "> 4 years" got better results on functional tests than the group of illiterates. This study showed that the patients with higher PLA levels had higher scores on cognitive tests than the participants with lower PLA levels. We found that participants who belonged to the "Intermediate level professions, intellectual and scientific activity" group achieved higher results on cognitive tests than those that belonged to the "Unskilled workers" group, corroborating the findings of other studies ${ }^{31}$ that showed that substantively complex work significantly increased the level of intellectual functioning. The participants with higher CR levels had higher scores on cognitive tests than elderly with lower CR levels.

The main limitation of this study was the small sample size, the small variability of education level, the fact that some tests are not validated for the Portuguese population and particularly the study design was a cross-sectional observation. Future studies with larger sample sizes will be needed to address these limitations and we must design prospective long-term follow-up observations.

In conclusion, we must highlight that the level of $\mathrm{CR}$ influenced the severity of $\mathrm{AD}$. According to Tucker and Stern $^{5}$, CR explains why those with higher IQ, education, occupational attainment, or PLA, evidence less severe clinical or cognitive changes in the presence of age-related or $\mathrm{AD}$ pathology.

CR is not fixed and continues to evolve across the lifespan. We agree that there are advantages in achieving high levels of CR and we think that healthy life styles should be promoted to improve the health of older adults, helping them to make healthy choices, such as lifelong cognitive stimulation, maintaining an active life or getting regular physical activity. Community preventive approaches for dementia should focus on the improvement of education, changes in lifestyles and the promotion of PLA.

This study suggests that $\mathrm{AD}$ patients with a higher $\mathrm{CR}$ may benefit against cognitive decline after diagnosis of $\mathrm{AD}$. However, will be needed longitudinal studies to clarify the course of $\mathrm{AD}$ along different stages of the disease and its relationship with CR.

\section{References}

1. Berr C, Wancata J, Ritchie K. Prevalence of dementia in elderly in Europe. European Neuropsychopharmacol. 2005;15(4):463-71. http://dx.doi.org/10.1016/j.euroneuro.2005.04.003

2. Siedlecki K, Stern Y, Reuben A, Sacco RL, Elkind MSV, Wright CB. Construct validity of cognitive reserve in multiethnic cohort: The Northen Manhattan Study. J Int Neuropsychol Soc. 2009;15(4):558-69. http://dx.doi.org/ 10.1017/S1355617709090857
3. Scarmeas N, Albert SM, Manly JJ, Stern Y. Education and rates of cognitive decline in incident Alzheimer's disease. J Neurol Neurosurg Psychiatric. 2006;77(3):308-16. http://dx.doi.org/10.1136/jnnp.2005.072306

4. Stern Y. Cognitive reserve in ageing and Alzheimer's disease. Lancet Neurol. 2012;11(11):1006-12. http://dx.doi.org/10.1016/S1474-4422(12)70191-6. 
5. Tucker AM, Stern Y. Cognitive reserve in aging. Curr Alzheimer Res. 2011;8(4):354-60.

6. Stern Y. Cognitive reserve: implications for assessment and interventions. Folia Phoniatr Logop. 2013;65:49-54. http://dx.doi.org/10.1159/000353443.

7. Sobral M, Paúl C. Education, leisure activities and cognitive and functional ability of Alzheimer's disease patients: a follow-up study. Dement Neuropsychol. 2013;7(2):181-9.

8. Tschanz JT, Corcoran CD, Schwartz S, Treiber K, Green RC, Norton $\mathrm{MC}$ et al. Progression of cognitive, functional and neuropsychiatric symptom domains in a population cohort with Alzheimer's dementia the cache county dementia progression study. Am J Geriatr Psychiatry. 2011;19(6):532-42. http://dx.doi.org/10.1097/JGP.0b013e3181faec23.

9. Helzner E, Scarmeas N, Cosentino S, Portet F, Stern Y. Leisure activity and cognitive decline in incident Alzheimer disease. Arch Neurol. 2007;64(12):1749-54. http://dx.doi.org/10.1001/archneur.64.12.1749

10. Wilson RS, Li Y, Aggarwal NT, Barnes LL, McCann JJ, Gilley DW et al. Education and the course of cognitive decline in Alzheimer. Neurology. 2004;63(7):1193-202. http://dx.doi.org/10.1212/01.WNL.0000140488.65299.53

11. Fritsch T, McClendon MJ, Smyth K, Ogrocki PK. Effects of educational attainment and occupational status on cognitive and functional decline in persons with Alzheimer-type dementia. Internat Psychogeriatr. 2002;14:347-63. http://dx.doi.org/10.1017/S1041610202008554

12. Teiber KA, Carlson MC, Corcoran $\mathrm{C}$ et al. Cognitive stimulation and cognitive and functional decline in Alzheimer's disease: the cache county dementia progression study. J Gerontol B Psychol Sci Soc Sci. 2011;66 (4):416-25. http://dx.doi.org/10.1093/geronb/gbr023

13. Katzman R, Brown T, Thal LJ, Fuld PA, Aronson M, Butters $\mathrm{N}$ et al. Comparison of rate of annual change of mental status score in four independent studies of patients with Alzheimer's disease. Ann Neurol. 1988;24(3):384-9. http://dx.doi.org/10.1002/ana.410240306

14. American Psychiatric Association. Diagnostic and statistical manual of mental disorders (DSM-5). 5th ed. Washington, DC: American Psychiatric Association; 2013.

15. McKhann GM, Knopman DS, Chertkow H, Hyman BT, Jack Jr CR, Kawas $\mathrm{CH}$ et al. The diagnosis of dementia due to Alzheimer's disease: recommendations from the National Institute on Aging-Alzheimer's Association workgroups on diagnostic guidelines for Alzheimer's disease. Alzheimer's Dementia. 2011;7(3):263-9. http://dx.doi.org/10.1016/j.jalz.2011.03.005

16. Rami L, Valls-Pedret C, Bartrés-Faz D, Caprile C, Solé-Padullés C, Olives J, Bosch B et al. Cognitive reserve questionnaire: scores obtained in a healthy elderly population and in one with Alzheimer's disease. Rev Neurol. 2011;52(4):195-201. Spanish.

17. Sobral M, Pestana MH, Paúl C. Measures of cognitive reserve in Alzheimer's disease. Trends Psychiatry Psychother. 2014;36(3):160-8. http://dx.doi.org/10.1590/2237-6089-2014-0012
18. Folstein MF, Folstein SE, McHugh R. Mini-mental state: a practical method for grading the cognitive state for patients for the clinician. J Psychiatric Res. 1975;12(3):189-98. http://dx.doi.org/10.1016/0022-3956(75)90026-6

19. Guerreiro M., Silva AP, Botelho M., Leitão O, Castro-Caldas A, Garcia C. Adaptação à população portuguesa da tradução do Mini Mental State Examination (MMSE). Rev Port Neurologia. 1994;1:9.

20. Hughes CP, Berg L, Danzinger LW, Coben LA, Martin RL. A new clinical scale for the staging of dementia. Brit J Psychiatry. 1982;140:566-72. http://dx.doi.org/10.1192/bjp.140.6.566

21. Garret C, Santos F, Tracana I, Barreto J, Sobral M, Fonseca R. Avaliação clínica da demência. In: Grupo de Estudos de Envelhecimento Cerebral e Demências, editor. Escalas e testes na demência. Lisboa: Grupo de Estudos de Envelhecimento Cerebral e Demências; 2008. p. 17-32.

22. Miosh E, Dawson K, Mitchell J, Arnold R, Hodges JR. The Addenbrooke's Cognitive Examination Revised (ACE-R): a brief cognitive test battery for dementia screening. Int J Geriatr Psychiatry. 2006;21(11):1078-85. http://dx.doi.org/10.1002/gps.1610

23. Simões MR, Pinho MS, Prieto G et al. Addenbrooke's Cognitive Examination Revised (ACE-R). In: Mário R, Simões, Santana I, editores. Escalas e testes na demência. 3a ed. Lisboa: Novartis; 2014. p. 26-31.

24. Mahoney FI, Barthel DW. Functional evaluation: the Barthel index. Md State Med J. 1965;14:61-5.

25. Lawton MP, Brody EM. Assessment of older people: self-maintaining and instrumental activities of daily living. Gerontologist. 1969;9(3):179-86.

26. Graffar M. Une méthode de classification sociale d'échantillons de population. Courier. 1956;6:445-9.

27. Instituto Nacional de Estatística. Classificação nacional de profissões 2010 Lisboa: National Statistical Institute; 2011.

28. Pestana MH, Gageiro JN. Análise categórica, árvores de decisão e análise de conteúdo em ciências sociais e da saúde com o SPSS. Lisboa: Lidel; 2009. ISBN 978-972-757-585-5.

29. Prince M, Bryce R, Albanese E, Wimo A, Ribeiro W, Ferri CP. The global prevalence of dementia: a systematic review and metaanalysis. Alzheimers Dement. 2013;9(1):63-75. http://dx.doi.org/10.1016/j.jalz.2012.11.007

30. Ganguli M, Snitz BE, Lee CW, Vanderbilt J, Saxton JA, Chang CC. Age and education effects and norms on a cognitive test battery from a population-based cohort: the Monongahela-Youghiogheny Healthy Aging Team. Aging Mental Health. 2010;14(1):100-7. http://dx.doi.org/10.1080/13607860903071014

31. Schooler C, Mulatu MS, Oates G. The continuing effects of substantively complex work on the intellectual functioning of older workers. Psychology Aging. 1999;14(3):483-506. http://dx.doi.org/10.1037/0882-7974.14.3.483 